\title{
A COMPARATIVE STUDY OF PERINATAL OUTCOME IN LOW RISK PREGNANCIES WITH CTG MONITORING AND INTERMITTENT AUSCULTATION
}

\author{
Velimala Ratna Kumari ${ }^{1}$, Indiramani $^{2}$, Kousalya Chakravarthy ${ }^{3}$, A. Anitha $^{4}$ \\ 1 Professor and Chief, Department of Obstetrics and Gynaecology, Niloufer Hospital for Women and Children, Osmania Medical College, \\ Hyderabad. \\ ${ }^{2}$ Assistant Professor, Department of Obstetrics and Gynaecology, Niloufer Hospital for Women and Children, Osmania Medical College, \\ Hyderabad. \\ ${ }^{3}$ Assistant Professor, Department of Anaesthesia, Niloufer Hospital for Women and Children, Osmania Medical College, Hyderabad. \\ ${ }^{4}$ Post Graduate, Department of Obstetrics and Gynaecology, Osmania Medical College, Hyderabad.
}

\section{ABSTRACT}

EFM was introduced into widespread clinical practice in the 1970s to 1980s on the premise that it would facilitate early detection of abnormal FHR patterns thought to be associated with hypoxia thus allowing earlier intervention to prevent foetal neurological damage and/or death. There is a lack of evidence of benefit supporting the use of the admission CTG in low-risk pregnancy. In this study we the aim to evaluate the effects of Cardiotocograph Foetal Monitoring on perinatal outcome in low risk Obstetric population and determine the cost effective and reliable method of fetal monitoring that is applicable to low-risk population.

\section{METHODOLOGY}

A prospective randomized study conducted on 200 low risk pregnant women in labour divided into 2 groups of 100 each. Group A includes those monitored with admission CTG and Group B includes those monitored with intermittent auscultation (IA).

\section{OBSERVATION AND RESULTS}

The demographic features, parity and gestational age in both the groups were comparable; 10 out of the 100 in CTG group had meconium stained liquor whereas 15 of them had meconium in IA group; 71\% of the patients in CTG group had normal delivery, whereas it was $84 \%$ in IA group. Incidence of LSCS was $23 \%$ in CTG group as against 9\% in IA group. A 'P' value of 0.02, RR of 25 for operative deliveries in CTG group was observed which was significant. Incidence of AVD was 6\% in CTG group and 7\% in IA group with a $p$ value of $<0.05$, which is statistically significant. The incidence of MSL, APGAR scores at 1,5 and 10 minutes and NICU admissions were comparable in both the groups. There was no significant difference in babies with low APGAR $<7$ at 5 min and NICU admissions in both the groups. In our study the sensitivity of CTG was $63.63 \%$, specificity $80.35 \%$, positive predictive value $33.3 \%$, negative predictive value $94.93 \%$. The low sensitivity and high false positives led to the intervention in delivery and increase in operative delivery with no difference in perinatal outcome.

\section{CONCLUSION}

Use of Cardiotocograph monitoring in low risk pregnancies has no better role than intermittent Auscultation in improving perinatal outcome. CTG monitoring in low risk pregnancies caused a significant rise in Caesarean deliveries, which could have otherwise been avoided. It is time that practice guidelines for intermittent auscultation be used, promoted, and published extensively in the obstetric world, so that caesarean rates may be dramatically decreased, and women's needs may be honoured by those attending her birth.

\section{KEYWORDS}

Cardiotocograph (CTG), Intermittent Auscultation (IA), Low Risk Pregnancies.

HOW TO CITE THIS ARTICLE: Kumari VR, Indiramani, Chakravarthy K, et al. "A Comparative study of perinatal outcome in low risk pregnancies with CTG monitoring and intermittent auscultation." Journal of Evolution of Medical and Dental Sciences 2015; Vol. 4, Issue 105, December 31; Page: 17038-17042, D0I: 10.14260/jemds/2015/2579

\section{INTRODUCTION}

CTG was introduced as a means of attempting to identify those fetuses of low-risk mothers at greatest risk of intrapartum hypoxia (Arul Kumaran 2000: RCOG 2001).[1] who might benefit from more intensive monitoring by continuous EFM and/or fetal scalp blood gas analysis or from immediate intervention (e.g. expedited birth).

Financial or Other, Competing Interest: None.

Submission 07-12-2015, Peer Review 08-12-2015,

Acceptance 26-12-2015, Published 31-12-2015.

Corresponding Author:

Dr. Kousalya Chakravarthy,

Assistant Professor,

Department of Anaesthesia.

Niloufer Hospital for Women \& Children,

Osmania Medical College.

Hyderabad.

E-mail:dr.kausi.c@gmail.com

DOI:10.14260/jemds/2015/2579
The concept of Electronic foetal monitoring by was received with huge fanfare and optimism and recognized as an obstetrician's window into the complex interplay of intrapartum events complications, suboptimal uterine perfusion, placental dysfunction which can result in adverse neonatal outcome.[2,3] Hence it was fast adopted into most of the maternity units worldwide, virtually rendering the intermittent auscultation a dying skill today.

There is a lack of evidence of benefit supporting the use of the admission CTG in low-risk pregnancy. Despite recommendations that it should not be recommended for this group of women.[4,5] and existing controversy about nomenclature, inter observer and intra observer variability, inadequate training and without guidelines for management algorithms the admission CTG is still widely used. 


\section{AIM OF THE STUDY}

To determine whether intrapartum admission Cardiotocography (CTG) in women at low obstetric risk, is associated with improved perinatal natal outcome and to analyse whether it increases the incidence of instrumental delivery and caesarean section. The study was undertaken to determine the Sensitivity, Specificity and Predictive value of CTG as a screening test.

\section{METHODOLOGY}

The present study is a Randomized prospective study done in the Department of Obstetrics and Gynaecology at Niloufer hospital, a tertiary care teaching hospital [Annual statistics is about 6000 deliveries] for a period of one year during Nov 2013 to Oct 2014. A total of 200 low-risk pregnant women who reported to labour ward with signs of possible labour were randomly selected.

The women were randomly allocated into 2 groups of 100 each. Group A includes those monitored with admission CTG and Group B includes those monitored with Intermittent Auscultation (IA). All the pregnant patients with singleton pregnancies in cephalic presentation with gestational age 3742 wks. and in spontaneous labour are included in the study. Those patients with multiple pregnancies scar on the uterus, noncephalic presentations, PROM, preterm or post term pregnancies, induced labour, APH, ultrasound confirmed foetal congenital anomalies and IUGR are excluded from the study.

\section{PROCEDURE IN DETAIL}

A detailed history was taken at the admission which includes age, parity, and date of last menstrual period social, occupational and economic class. Relevant family history of genetic diseases, incidence of congenital anomalies in the family, personal history like abuse of toxic substances etc. are taken to rule out possible high risk factors [International guidelines for intapartum care.

Informed consent was taken after explaining the procedure in detail. A Labour Admission Test [LAT] was done for $20 \mathrm{~min}$ after initial assessment to document vital signs, obstetric examination to confirm the fetal lie, presentation, station, cervical dilatation and status of membranes.

A baseline CTG trace was taken with the patient in semi recumbent position. Our study was confined to CTG trace. The other components of Electronic Foetal Monitoring (EFM) like foetal pulse oximetry, foetal ECG etc. are not included. The initial CTG trace was interpreted as per ACOG Practice Bulletin 2009 guidelines.

Those with Category 1 trace are allocated randomly into Intermittent CTG group (Group A) or cross over to IA (Group $B)$. The patients allocated into Group B are monitored by Intermittent Auscultation (IA) method with Protocol as per RCOG 2001 Guidelines. The results were compared with Chi square test. A p value of $<0.5$ was considered statistically significant

\section{OBSERVATION AND RESULTS}

The demographic data in both the groups were comparable (Table1). The duration of CTG in the LAT (Labour Admission Test) was 20 minutes in 94 patients. 6 out of the 100 patients needed prolonged CTG monitoring. Of the CTGs 79 belonged to Category 1,7 to category 2 and 14 to category 3. During
Intrapartum period 10 patients in CTG group and 15 in IA group developed meconium stained liquor. The mode of delivery was SVD (Spontaneous vaginal delivery) in 71 of CTG group and 84 in IA group, AVD in 6 patients of CTG group versus 7 in IA group.

The rate of Emergency LSCS was much higher in CTG group with 23 emergency caesarean sections as versus 9 in IA group. Both AVD and emergency caesarean sections were more in CTG group. Presumed foetal compromise was the most common indication for intervention in the CTG group (6 AVDs and 10 LSCS), where as in IA group the intervention for foetal compromise was comparatively less (4 AVDs and 1 LSCS) (Table 2). The difference in the interventional deliveries was statistically significant with a p value of 0.02 and a RR of 2.5 .

\section{Neonatal outcome}

The birth weights of the new-borns in both the groups were comparable and not statistically significant. The A p value of 0.05 was observed for Low APGAR scores, which was not significant and a ' $\mathrm{P}$ ' value of 0.7 was observed for NICU admissions, which was again not significant. There was no statistical significant difference in the incidence of MSL in both the groups (Table 3).

\section{Interpretation of CTG}

Seventy nine of the CTGs belonged to Category 1, 7 to category 2 and 14 were of category 3. Statistical analysis revealed 7 True Positives, 14 False Positives, 4 False Negatives and 75 True negatives. In our study the sensitivity of CTG was $63.63 \%$, specificity $80.35 \%$, positive predictive value $33.3 \%$ negative predictive value $94.93 \%$ (Table 4 ).

\begin{tabular}{|c|c|c|c|}
\hline \multirow{2}{*}{ Parameter } & & $\begin{array}{c}\text { CTG } \\
\text { Group }\end{array}$ & IA Group \\
\hline \multirow{3}{*}{ Age in years } & $<20$ & 5 & 12 \\
\cline { 2 - 4 } & $20-25$ yrs & 72 & 76 \\
\cline { 2 - 4 } & $>25$ yrs & 23 & 12 \\
\hline \multirow{2}{*}{$\begin{array}{c}\text { Pregnancy } \\
\text { status }\end{array}$} & Nulliparous & 56 & 59 \\
\cline { 2 - 4 } & Multiparous & 44 & 41 \\
\hline \multirow{2}{*}{ Gestational age } & Early term & 24 & 32 \\
\cline { 2 - 4 } & Late term & 76 & 68 \\
\hline \multicolumn{3}{|c|}{ Table 1: Demographic Features } \\
\hline
\end{tabular}

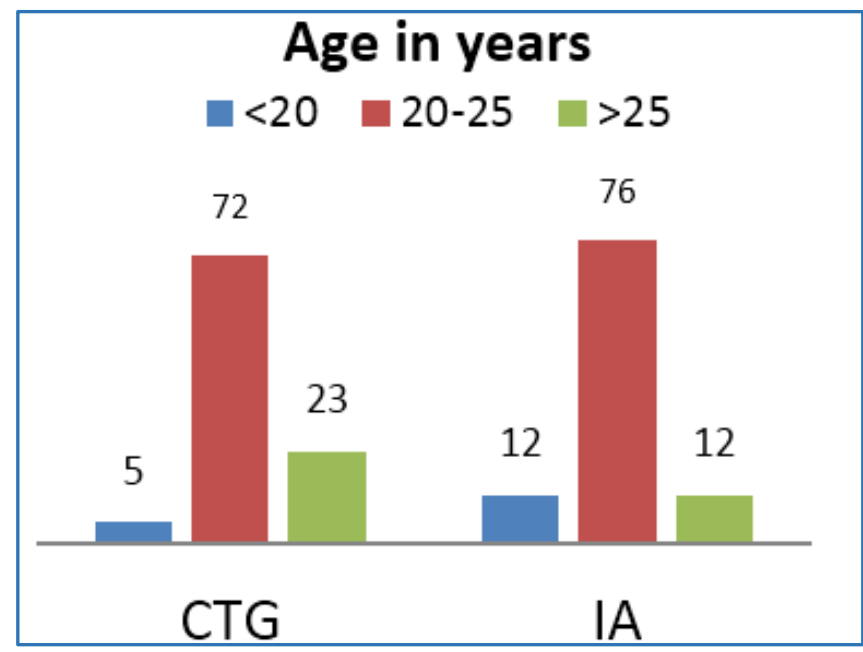



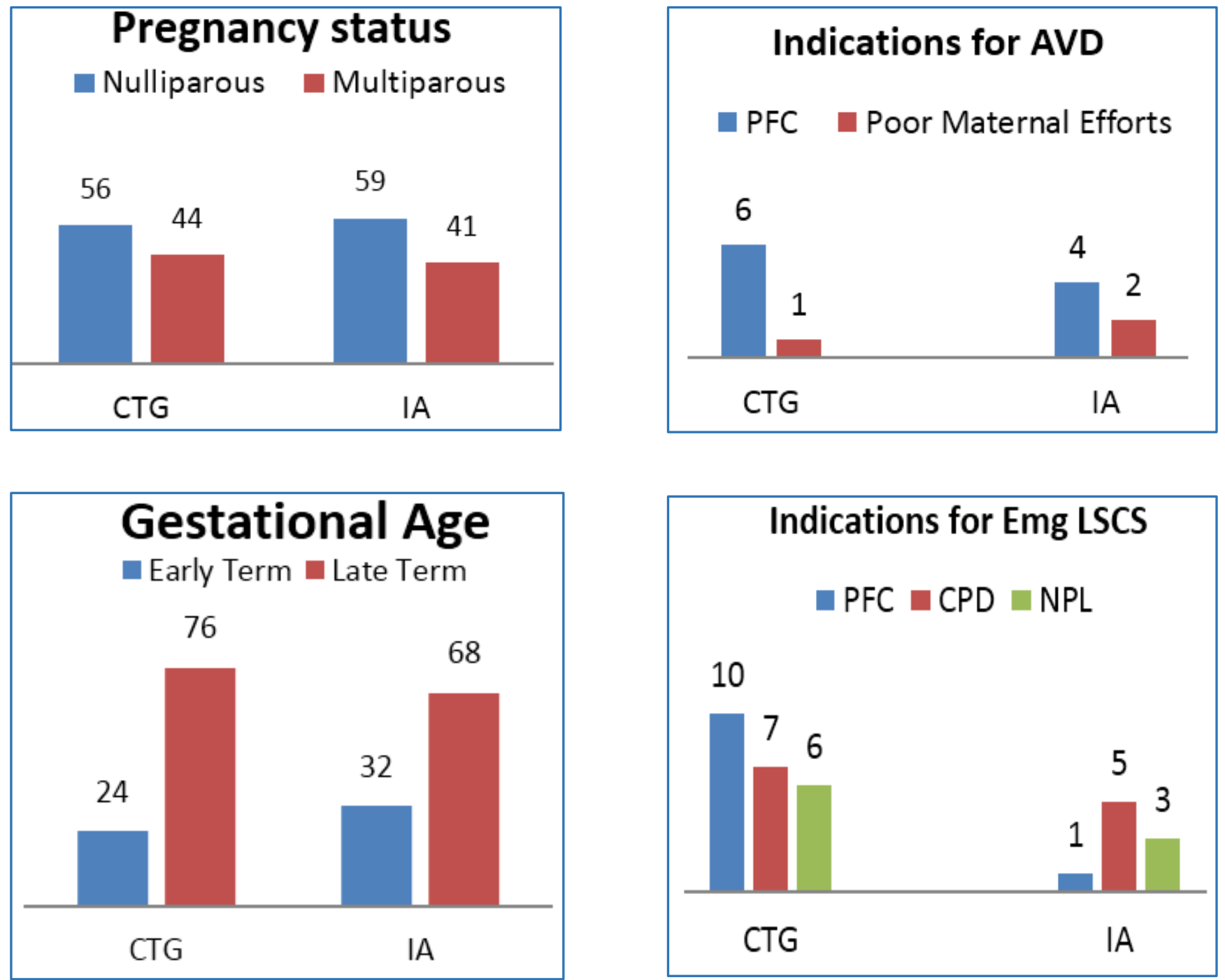

\begin{tabular}{|c|c|c|}
\hline Indications for AVDs (Outlet Forceps) & CTG & IA \\
\hline Presumed fetal compromise & 6 & 4 \\
\hline Poor maternal efforts & 1 & 2 \\
\hline Indications for Emergency LSCS & CTG & IA \\
\hline Presumed fetal compromise & 10 & 1 \\
\hline CPD & 7 & 5 \\
\hline Non progression of labour & 6 & 3 \\
\hline Table 2: Indications for intervention in Deliveries \\
\hline
\end{tabular}

\begin{tabular}{|c|c|c|c|c|c|c|c|c|c|}
\hline \multicolumn{2}{|c|}{ Sl. No. } & Parameters & CTG & $\%$ & IA & $\%$ & P Value & R.R & Significance \\
\hline \multicolumn{2}{|c|}{1} & Total cases(n) & 100 & $100 \%$ & 100 & $100 \%$ & & & \\
\hline \multicolumn{2}{|c|}{2} & Spontaneous Vaginal Deliveries & 71 & $71 \%$ & 84 & $84 \%$ & 0.12 & & $\mathrm{~S}$ \\
\hline \multicolumn{2}{|c|}{3} & Emergency LSCS & 23 & $23 \%$ & 9 & $9 \%$ & 0.02 & 2.5 & $\mathrm{~S}$ \\
\hline \multicolumn{2}{|c|}{4} & AVDs (Forceps) & 7 & $7 \%$ & 6 & $6 \%$ & 0.02 & 2.5 & $\mathrm{~S}$ \\
\hline \multicolumn{2}{|c|}{5} & Birth weight & & & & & & & \\
\hline & & $<2 \mathrm{~kg}$ & 4 & $4 \%$ & 1 & $1 \%$ & & & NS \\
\hline & & $2-2.5 \mathrm{~kg}$ & 75 & $75 \%$ & 71 & $71 \%$ & & & NS \\
\hline & & $>2.5 \mathrm{~kg}$ & 21 & $21 \%$ & 28 & $28 \%$ & & & NS \\
\hline \multicolumn{2}{|c|}{6} & Low APGAR & & & & & & & \\
\hline & $\mathrm{A}$ & $<7$ at $1 \mathrm{~min}$ & 7 & $1 \%$ & 11 & $6 \%$ & 0.07 & 0.9 & NS \\
\hline & $\mathrm{B}$ & $<7$ at $5 \mathrm{~min}$ & Nil & Nil & 4 & $4 \%$ & 0.07 & & NS \\
\hline & $\mathrm{C}$ & $<4$ at $10 \mathrm{~min}$ & Nil & Nil & 2 & $1 \%$ & 0.12 & & NS \\
\hline \multirow{4}{*}{7} & & NICU Admissions & 12 & $11 \%$ & 14 & $14 \%$ & 0.7 & 0.9 & NS \\
\hline & $\mathrm{A}$ & $<3$ days & 6 & $6 \%$ & 8 & $8 \%$ & 0.7 & & NS \\
\hline & $\mathrm{B}$ & 3-7 days & 6 & $6 \%$ & 5 & $5 \%$ & 0.07 & 1.2 & NS \\
\hline & $\mathrm{C}$ & $>7$ days & 0 & & 1 & & 0.7 & & NS \\
\hline 8 & & MSL & 10 & $10 \%$ & 15 & $15 \%$ & & 0.6 & NS \\
\hline
\end{tabular}




\begin{tabular}{|c|c|c|c|}
\hline Category of CTG & $\begin{array}{c}\text { Cat } 1 \\
79\end{array}$ & $\begin{array}{c}\text { Cat } 2 \\
07\end{array}$ & $\begin{array}{c}\text { Cat } 3 \\
14\end{array}$ \\
\hline Newborn with evidence of distress & $\begin{array}{c}\mathrm{TP} \\
7\end{array}$ & $\begin{array}{c}\text { FN } \\
4\end{array}$ & 11 \\
\hline Without distress & $\begin{array}{l}\text { FP } \\
14\end{array}$ & $\begin{array}{l}\mathrm{TN} \\
75\end{array}$ & 89 \\
\hline & 21 & 79 & 100 \\
\hline Sensitivity of CTG & \multicolumn{2}{|c|}{$\mathrm{TP} / \mathrm{TP}+\mathrm{FN}=7 / 7+4$} & $63.63 \%$ \\
\hline Specificity of CTG & \multicolumn{2}{|c|}{$\mathrm{TN} / \mathrm{FP}+\mathrm{TN}=75 / 14+75$} & $80.35 \%$ \\
\hline Positive Predictive Value (PPV) & \multicolumn{2}{|c|}{$\mathrm{TP} / \mathrm{TP}+\mathrm{FP}=7 / 7+14$} & $33.3 \%$ \\
\hline Negative Predictive Value (NPV) & \multicolumn{2}{|c|}{$\mathrm{TN} / \mathrm{TN}+\mathrm{FN}=75 / 75+4$} & $94.93 \%$ \\
\hline \multicolumn{4}{|c|}{$\begin{array}{c}\text { Table 4: Statistical significance of CTG in predicting foetal compromise } \\
\text { (Sensitivity, Specificity, PPV and NPV of CTG) }\end{array}$} \\
\hline
\end{tabular}

\section{DISCUSSION}

There is wide intra observer and inter observer variation in the interpretation of cardiotocograms even among experts. Fetal heart variability is difficult to interpret visually, and there is a tendency to over report abnormalities.[6] A high percentage of admission cardiotocograms were reported as abnormal, with reduced variability and variable decelerations the most commonly reported abnormalities.

Variability in fetal heart rate cannot be assessed in the Doppler group. When an admission cardiotocogram shows possible reduced baseline variability or mild decelerations, midwives and obstetricians will take defensive action. This starts with continuous monitoring of fetal heart rate, which leads to increased obstetric intervention in the form of augmentation of labour, epidural analgesia, and ultimately increased rates of obstetric interventions.

In our study after LAT (Labour Admission Test).[7] for 20 minutes 6 out of 100 cases needed continuous monitoring. 5 out of them had Emergency LSCS and 1 had outlet forceps delivery; 4 of the 6 new-borns needed NICU admissions. We opine that LAT is important to define low risk pregnancy and the duration of LAT may have an impact on the perinatal outcome.

In the CTG group a total of $7+14$ babies were identified as presumed foetal compromise as evidenced by Category 2 and Category 3 CTG traces respectively. Among them only 6 new-borns needed NICU admission. A high amount of false positives were identified, which led to the intervention in delivery; $2^{\text {nd }}$ stage of labour, inadequate contact between maternal abdomen and the transducer, inexperience of the staff in interpreting CTG has led to high false positives and unnecessary obstetric interventions.

False negatives were identified in 4 of them. Failure to detect abrupt changes after the normal Admission Test, Prolonged Admission Delivery Interval may have contributed to high false negatives.[8]

In our study the sensitivity of CTG in predicting compromised foetus was $63.63 \%$, specificity $80.35 \%$, positive predictive value $33.3 \%$ negative predictive value $94.93 \%$. With a low Sensitivity of $63.63 \%$ and a poor Specificity of $80.35 \%$, the CTG may not be a very reliable Screening test for identifying foetuses at risk of developing intrapartum hypoxia.

It is established that majority of cases of cerebral palsy in non-anomalous term infants are not associated with intrapartum hypoxia-ischemia. As only $10-14.5 \%$ of proportion of cerebral palsy is associated with intrapartum hypoxia-ischemia the role of CTG decreasing the incidence of cerebral palsy is limited.[9]

Moreover it resulted in unnecessary increase in interventions and was instrumental in some women for needing continuous monitoring with no improved outcomes. Our study coincides with the studies by Gourounti et al.[10,11] Wood et al.[12] and Kelso et al.[13] where they have found that routine electronic fetal monitoring in labour results in increased unnecessary intervention for fetal compromise (Table 5).

\begin{tabular}{|c|c|c|c|c|}
\hline OUTCOME VARIABLE & $\begin{array}{l}\text { GOUROUNTI AND SANDALL } \\
\text { [2006 MIRES, CHEYNE, } \\
\text { IMPEY }\end{array}$ & $\begin{array}{l}\text { WOOD et al. } \\
\text { 1981] Melbourne }\end{array}$ & $\begin{array}{c}\text { KELSO et al. } 1978 \\
\text { Sheffield }\end{array}$ & PRESENT STUDY \\
\hline No. of subjects & 850 low risk women & $\begin{array}{l}828 \text { low-risk } \\
\text { women }\end{array}$ & $\begin{array}{l}504 \text { low risk } \\
\text { women }\end{array}$ & 100 low risk women \\
\hline Incidence of ISCS & Pooled RR 1.2\% & No difference & Significant $\mathrm{p}<0.05$ & Significant $\mathrm{p}<0.02$ \\
\hline $\begin{array}{c}\text { Assisted vaginal } \\
\text { deliveries }\end{array}$ & Pooled RR 1.1\% & Significant $\mathrm{p}<0.01$ & NS & Significant $\mathrm{p}<0.02$ \\
\hline Low Apgar < 7AT 1 min & $1.25 \% 1.25 \%$ & NS & NS & NS \\
\hline Low Apgar $<4$ at $5 \mathrm{~min}$ & & NS & NS & NS \\
\hline NICU Admissions & & $\begin{array}{l}\text { I perinatal death } \\
\text { in monitored } \\
\text { group } \\
\end{array}$ & & NS \\
\hline Conclusion & $\begin{array}{c}\text { No significance of NICU } \\
\text { admissions in both groups, } \\
\text { but incidence of CS is high in } \\
\text { CTG group }\end{array}$ & & $\begin{array}{l}\text { Neither beneficial } \\
\text { nor harmful to use } \\
\text { CTG }\end{array}$ & $\begin{array}{l}\text { Increase in operative } \\
\text { with no difference in } \\
\text { perinatal outcome }\end{array}$ \\
\hline
\end{tabular}


The popular study conducted at Dublin [14] reviewed women with a highly abnormal CTG in labour, i.e. fetal tachycardia with reduced variability and late decelerations. They found that only $58 \%$ of these fetuses were acidotic at birth as judged by umbilical artery $\mathrm{pH}$ and only $0.2 \%$ went on to develop CP. Due to this high false positive rate continuous FHR monitoring, cannot be recommended as a predictor of CP.

The current recommendation of intermittent auscultation every $15 \mathrm{~min}$ in the first stage can be modified to increase the compliance and lessen the unnecessary burden, stress and medico-legal liability for birth attendants. Sholapurkar.[15] recommends intermittent auscultation for 60 seconds before and after three contractions over about $10 \mathrm{~min}$ every half an hour in the first stage of labour. This could be more practical and patient friendly and at the same time could improve detection of fetal distress.[15]

\section{CONCLUSION}

Use of Cardiotocograph monitoring in low risk pregnancies has no better role than Intermittent Auscultation in improving perinatal outcome. Routine electronic fetal monitoring in labour results in increased unnecessary intervention for fetal compromise. Structured Intermittent Auscultation is equally efficacious to CTG monitoring in improving the perinatal outcome in low risk pregnancies. It is time that practice guidelines for intermittent auscultation be used, promoted, and published extensively in the obstetric world, so that cesarean rates may be dramatically decreased, and women's needs may be honoured by those attending her birth.

\section{REFERENCES}

1. Donald Gibb, Sabaratnam Arulkumaran. Fetal Monitoring in Practice, 3rd Edition Oxford Elseiver Churchill Livingstone Title ISBN: 978-0-443-10004-8.

2. Nelson KB, Ellenberg JH. Obstetric complications as risk factors for cerebral palsy or seizure disorders. JAMA. 1984 Apr 13;251(14):1843-8.

3. Nelson KB, Ellenberg JH. Antecedents of cerebral palsy. Multivariate analysis of risk. N Engl J Med 1986 Jul 10;315(2):81-6.

4. Hon EH, Lee ST. Electronic evaluation of the fetal heart rate. Patterns preceding fetal death, further observations. Am J Obstet Gynecol. 1963 Nov 15;87:81426.

5. Raymond G Kennedy. Electronic Fetal Heart Rate Monitoring: Retrospective Reflections on a TwentiethCentury Technology. J R Soc Med May 1998 vol. 91 no. 5244-250.
6. Schiermeir $\mathrm{S}$, Westhof $\mathrm{G}$, Leven $\mathrm{A}$, et al. Intra- and interobserver variability of intrapartum cardiotocography: A multicenter study comparing the FIGO classification with computer analysis software. Gynecol Obstet Invest. 2011;72(3):169-73.

7. Ingemarsson I, Arulkumaran S, Ingemarsson $\mathrm{E}$, et al. Admission test: a screening test for fetal distress in labour. Obstet Gynecol. 1986 Dec;68(6):800-6.

8. Faridah Hanim Zam Zam, Nazimah Idris, Tham Seng Who. Second stage fetal heart rate patterns and neonatal acid-base status. IeJSME 2012 6(2):18-23.

9. Graham EM ${ }^{1}$, Ruis KA, Hartman AL, et al. A systematic review of the role of intrapartum hypoxia-ischemia in the causation of neonatal encephalopathy. Am J Obstet Gynecol. 2008 Dec;199(6):587-95.

10. Gourounti $\mathrm{K}^{1}$, Sandall J. Admission cardiotocography versus intermittent auscultation of fetal heart rate: effects on neonatal Apgar score, on the rate of caesarean sections and on the rate of instrumental delivery--a systematic review. Int J Nurs Stud 2007 Aug;44(6):102935.

11. Gourounti K. Cochrane review finds that use of cardiotocograph on admission to the labour ward, rather than intermittent auscultation of the fetal heart rate, may increase risk of caesarean in low-risk women. Vid Based Nurs. 2012 Oct;15(4):106-7.

12. Wood C, Renou P, Oats J, Farrell E, Beischer N, Anderson J. A controlled trial of fetal heart rate monitoring in a lowrisk obstetric population. American Journal of Obstetrics and Gynecology. Volume 141, Issue 6, 1981, Pages 527534.

13. Kelso IM, Parsons RJ, Lawrence GF, Arora SS, Edmonds DK, Cooke ID. An assessment of continuous fetal heart rate monitoring in labour. A randomized trial. Am J Obstet Gynecol. 1978 Jul 1;131(5):526-32.

14. MacDonald D, Grant A, Sheridan-Pereira M, et al. The Dublin randomized controlled trial of intrapartum fetal heart rate monitoring. Am J Obstet Gynecol. 1985 Jul 1;152(5):524-39.

15. Sholapurkar SL. Intermittent auscultation of fetal heart rate during labour - a widely accepted technique for low risk pregnancies: but are the current national guidelines robust and practical? J Obstet Gynaecol. 2010;30(6):53740. 